\title{
ACONITINE-CONTAINING AGENT ENHANCES ANTITUMOR ACTIVITY OF DICHLOROACETATE AGAINST EHRLICH CARCINOMA
}

\author{
O.N. Pyaskovskaya, I.V. Boychuk, A.G. Fedorchuk, D.L. Kolesnik, O.I. Dasyukevich, G.I. Solyanik* \\ R.E. Kavetsky Institute of Experimental Pathology, Oncology and Radiobiology, NAS of Ukraine, Kyiv \\ 03022, Ukraine
}

\begin{abstract}
Significant variability of anticancer efficacy of dichloroacetate (DCA) stimulated an active search for the agents capable to enhance it antitumor action. Therefore, the aim of this work is the study of capability of aconitine-containing antiangiogenic agent $\mathrm{BC} 1$ to enhance anticancer activity of DCA against Ehrlich carcinoma. Materials and Methods: DCA (total dose was $1.3 \mathrm{~g} / \mathrm{kg}$ of b.w.) and BC1 (total dose was $0.9 \mathrm{mg} / \mathrm{kg}$ of b.w.) were administered per os starting from the $2^{\text {nd }}$ and $3^{\text {rd }}$ days, respectively $(8$ administrations for each agent). Antitumor efficacy of agents was estimated. Lactate level, LDH activity and the state of mitochondrial electron transport chain in tumor cells as well as phagocytic activity and reactive oxygen species (ROS) production of tumor-associated macrophages (TAM) were studied. Results: Combined administration of DCA and BC1 resulted in $89.8 \%$ tumor growth inhibition $(p<0.001)$, what is by $22.5 \%(p<0.05)$ higher that that of DCA alone. This combined treatment was accompanied with a decrease of lactate level in tumor tissue by $30 \%(\mathrm{p}<0.05)$ and significant elevation of LDH activity by $70 \%(\mathrm{p}<0.01)$. Increased level of NO-Fe-S clusters and 2-fold reduction of Fe-S cluster content were revealed in tumor tissue of mice after DCA and BC1 administration. It was shown that combined therapy did not effect TAM quantity and their phagocytic activity but stimulated ROS production by TAMs by $78 \%(p<0.05)$ compared to this index in control animals. Conclusion: Antiangiogenic agent BC1 in combination with DCA considerably enhances antitumor activity of DCA via significant decrease of Fe-S-containing protein level resulted from substantial elevation of nitrosylation of these proteins.
\end{abstract}

Key Words: Ehrlich carcinoma, dichloroacetate, aconitine-containing agent, tumor-associated macrophages, mitochondrial electron transport chain.

The achievements of recent years in regard to genetic mechanisms of cell metabolic regulation, mitochondrial functioning and synthesis of macromolecules have turn an attention to energy metabolism of tumor cells and Warburg effect [1]. Numerous metabolic pathways which are supposed to be important for tumor growth and progression are considered as perspective targets for the development of effective anticancer drugs $[2,3]$. Among such drugs dichloroacetate (DCA) could be mentioned $[4,5]$. It is known that DCA inhibits pyruvate dehydrogenase kinase what results in elevation of pyruvate dehydrogenase activity what in turn promotes the shift of cell metabolism from glycolysis toward oxidative phosphorylation, i.e. the state of tumor cells when their proliferative activity is decreased and apoptosis risk is enhanced [6, 7]. Apart from glycolysis inhibition, DCA may cause significant decrease of lactacidosis level in tumor cell microenvironment which is known to promote tumor cell viability [8].

For 30 years DCA has been used for correction of lacticemia, caused by high intensity of glycolysis or defective cell respiration. In 2008 Canadian scientists have shown that DCA is highly effective against many tumor types and possesses low toxicity toward normal tissues [9]. Such features of DCA have motivated an initiation of active scientific studies of this drug in oncology.

Submitted: May 18, 2015.

*Correspondence: E-mail: gsolyanik@gmail.com

Abbreviations used: DCA - dichloroacetate; LDH - lactate dehydrogenase; MtETC - mitochondrial electron-transport chain; ROS - reactive oxigen species; TAM - tumor-associated macrophages.
At present time, the data on anticancer efficacy of DCA are in large part contradictory. From one side, there are conclusive evidences obtained in experiments in vitro and in vivo on feasibility of DCA use as anticancer drug [10]. There has been documented an ability of DCA to suppress proliferation of many tumor cell lines with no impact on their death rates [6, 11]. Also, it has been shown that DCA induces apoptosis only at high doses and is active against cells with defective electron-transport chain [12, 13]. From other side, as it has been shown in preclinical studies DCA is ineffective against many experimental tumor models including lung cancer, neuroblastoma, lymphoma, sarcoma [14]. An analysis of the results of clinical studies performed in recent years has also revealed significant variability of anticancer efficacy of DCA: from its high efficacy to complete inefficacy $[15,16]$.

In the last years there is an active search for the agents capable to enhance anticancer action of DCA. Among such agents a special place is occupied by inhibitors of tumor angiogenesis providing the deficiency of the main energetic substrates (glucose and oxygen) via suppression of tumor vessel development [17].

Therefore, the aim of this work is the study of capability of antiangiogenic agent to enhance anticancer activity of DCA against Ehrlich carcinoma.

Aconitine-containing agent $\mathrm{BC} 1$ has been used as an inhibitor of tumor angiogenesis. As it has been shown earlier, $\mathrm{BC} 1$ exerts its antiangiogenic action via inhibition of endothelial cell proliferation which underlies its significant antitumor activity against tumors with angiogenesis-dependent growth [18, 19]. 


\section{MATERIALS AND METHODS}

Experimental animals and tumor strain. The study was carried out using 2.0-2.5 months white outbred mice weighting $18.5-21 \mathrm{~g}$, bred at animal facility of R.E. Kavetsky Institute of Experimental Pathology, Oncology and Radiobiology of the NAS of Ukraine (Kyiv, Ukraine). Animal study protocols and operation procedures were carried out in accordance with the main requirements to keeping and working with laboratory animals and to the rules of local Bioethics Committee.

Ehrlich carcinoma cells $\left(1.2 \cdot 10^{6}\right.$ cells in $0.2 \mathrm{ml}$ of physiological solution per animal) were transplanted subcutaneously in dorsal forelimb region.

Design of the study. After tumor cell transplantation the animals were distributed into 4 groups: control one and three experimental (Table 1). $0.4 \mathrm{ml}$ of aqueous DCA (Sigma, USA) solution were administered per os to animals from $1^{\text {st }}$ and $3^{\text {rd }}$ groups in a day starting from $2^{\text {nd }}$ day after tumor cell transplantation (8 administrations in total). The total DCA dose was $1.3 \mathrm{~g} / \mathrm{kg}$ of animal body weight. The mice from $3^{\text {rd }}$ group were also treated with aconitine-containing agent BC1 (Aksomed, Ukraine) each second day starting from $3^{\text {rd }}$ day after tumor cell transplantation ( 8 administrations in total). Total BC1 dose (by aconitine content) was $0.9 \mathrm{mg} / \mathrm{kg}$ of animal body weight. The animals from $2^{\text {nd }}$ group were administered with $\mathrm{BC} 1$ only, using the same dosage and regimen of administration as these for $3^{\text {rd }}$ group. Animals from control group received water for injections by the corresponding scheme.

Table 1. The design of experiment

\begin{tabular}{lcc}
\hline \multicolumn{1}{c}{ Groups of mice } & Number of mice/group & Type of treatment \\
\hline Control & 12 & $\mathrm{H}_{2} \mathrm{O}$ \\
Experimental-1 & 10 & $\mathrm{DCA}$ \\
Experimental-2 & 7 & $\mathrm{BC} 1$ \\
Experimental-3 & 10 & $\mathrm{DCA}+\mathrm{BC} 1$ \\
\hline
\end{tabular}

After tumor appearance its diameter has been measured triply per week using caliper. Tumor volumes were calculated by formula:

$$
V=3.14 \cdot d^{3} / 8
$$

where $d$ - tumor diameter.

Antitumor activity was evaluated at $17^{\text {th }}$ day after tumor cell transplantation with the use of tumor inhibition index $(D)$, calculated by the formula:

$$
D=\left(V_{k}-V\right) \cdot 100 \% / V_{k} \text {, }
$$

where $\mathrm{V}_{\mathrm{k}}$ and $\mathrm{V}$ - average tumor volumes in control and experimental groups, respectively.

At the $2^{\text {nd }}$ day after termination of the treatment (17 day of tumor growth) the animals were sacrificed under light ether narcosis. Tumor tissue was isolated. Then the effect of DCA on lactate level, lactate dehydrogenase (LDH) activity, functional state of mitochondrial electron-transport chain (MtETC), counts and functional activity of tumor-associated macrophages (TAM) have been analyzed. Each index has been evaluated in tumor tissue from 4-6 mice per each group.

Lactate level in tumor tissue was determined by spectrophotometry using LDH (Sigma, USA). The method is based on the reaction of lactate oxidation into pyruvate by $\mathrm{LDH}$ resulting in generation of $\mathrm{NADH}$ from NAD [20].

Total LDH activity in frozen tumor tissue was measured by spectrophotometric method which is based on determination of enzyme kinetics when pyruvate is converted to lactate [20].

For analysis of functional activity of MtETC in tumor cells, tumor tissue was cut into the samples of cylindrical shape $(d=4.0 \mathrm{~mm}, I=25-35 \mathrm{~mm})$, frozen and stored at $-70^{\circ} \mathrm{C}$. EPR analysis of the samples was performed at $77 \mathrm{~K}$ using spectrometer E-109 Varian (USA) at potential sweep speed of $500 \mathrm{E} / \mathrm{min}$, modulation amplitude of $1.25 \cdot 10 \mathrm{E}$, power of super-highfrequency radiation of $10.0 \mathrm{~mW}$, constant session of apparatus of $1.0 \mathrm{~s}$.

The levels of reduced non heme iron-sulfur (Fe-S) centers $(g=1.94)$ of MtETC proteins, nitrosyl (NO) complexes of heme iron $\left(g_{c e p}=2.01\right)$ and Fe-S nitrosyl complexes $\left(g_{c e p}=2.03\right)$ were determined by the data of EPR spectra.

Phagocytic activity (engulfing capacity) of TAM was determined by flow cytometer FACSCalibur (Becton Dickinson, USA). TAMs were identified with the use of anti-CD14 antibodies according to the instructions of the manufacturer (Becton Dickinson, USA). Analysis of phagocytic activity of $\mathrm{CD} 14^{+}$cells was performed with the use of FITC-labeled staphylococcus [21].

Reactive oxigenspecies (ROS) production by TAM was carried out using 2.7-dichlorodihydrofluorescein diacetate (Sigma, USA) [21] by flow cytometry.

Statistical analysis of the data was performed with the use of descriptive statistics, parametric Student's $t$-test and nonparametric Mann - Whitney U test, with the use of Microsoft Excel, Microcal Origin and Statistica. The data in the tables and figures are presented as mean \pm standard error.

\section{RESULTS AND DISCUSSION}

The performed studies have shown sufficiently high antitumor activity of DCA against Ehrlich carcinoma. The percent of tumor growth inhibition after the therapy $\left(17^{\text {th }}\right.$ day of tumor growth) achieved $73.3 \%$ (Table 2 ), what is in complete accordance with the results presented in the study [22].

Table 2. Antitumor efficacy of DCA, BC1 and their combination against Ehrlich carcinoma

\begin{tabular}{lcc}
\hline \multicolumn{1}{c}{ Groups of mice } & Tumor volume, $\mathrm{mm}^{3}$ & Inhibition index, \% \\
\hline Control & $7828.0 \pm 893.0$ & - \\
Experimental-1 & $2092.0 \pm 380.3^{*}$ & 73.3 \\
Experimental-2 & $5306.8 \pm 1595.3$ & - \\
Experimental-3 & $805.1 \pm 137.8^{*}$ & 89.8 \\
\hline
\end{tabular}
Note: ${ }^{*} \mathrm{p}<0.05$

In the group of animals treated with $\mathrm{BC} 1$, there was observed a tendency to inhibition of Ehrlich carcinoma growth which did not reach statistical significance due to variability of tumor volumes. This result is in agreement with our previous data on anticancer activity of $\mathrm{BC} 1$ against solid form of Ehrlich carcinoma. In this study growth kinetic analysis has shown that administration of $\mathrm{BC} 1$ (at the total dose of $0.9 \mathrm{mg} / \mathrm{kg}$ of b.w.) 
did not affect tumor volume till $15^{\text {th }}$ day and only after that tumor inhibition indices constantly increased growing up to $77.3 \%$ in a week after therapy completion [23].

Despite the fact that administration of antiangiogenic aconitine-containing drug $\mathrm{BC} 1$ to mice did not cause significant inhibition of tumor growth, introduction of this agent into DCA-based therapy significantly enhanced anticancer action of DCA. Combined administration of DCA and BC1 resulted in $89.8 \%$ tumor growth inhibition $(p<0.001)$, what is by $22.5 \%$ $(p<0.05)$ higher that that of DCA alone (see Table 2$)$.

Antitumor activity of DCA administered alone or in combination with $\mathrm{BC} 1$ was accompanied with statistically significant decrease of lactate level in tumor tissue by $22 \%(p<0.05)$ and $30 \%(p<0.05)$, respectively (Fig. 1, a), which may be caused by activation of DCAdependent oxidative decarboxylation of pyruvate and its conversion to acetyl-coenzyme A (the main substrate for Krebs cycle). Adaptive reaction of the cell on lactate level decrease is elevation of LDH activity; LDH activity increase by $120 \%(p<0.001)$ and $70 \%(p<0.01)$ was observed in mice treated with DCA and DCA + BC1, respectively (Fig. 1, $b$ ). Such activation of the enzyme could not compensate completely the decrease of lactate level at the background of DCA administration what could be possibly caused by higher rate of the pyruvate conversion to acetyl-coenzyme $A$ than to lactate. In the work [24] it has been shown that the DCA-dependent decrease of lactate level and increase of LDH activity in human colorectal cancer cells and prostate carcinoma cells has been accompanied with significant decrease of pyruvate-to-lactate exchange rate.
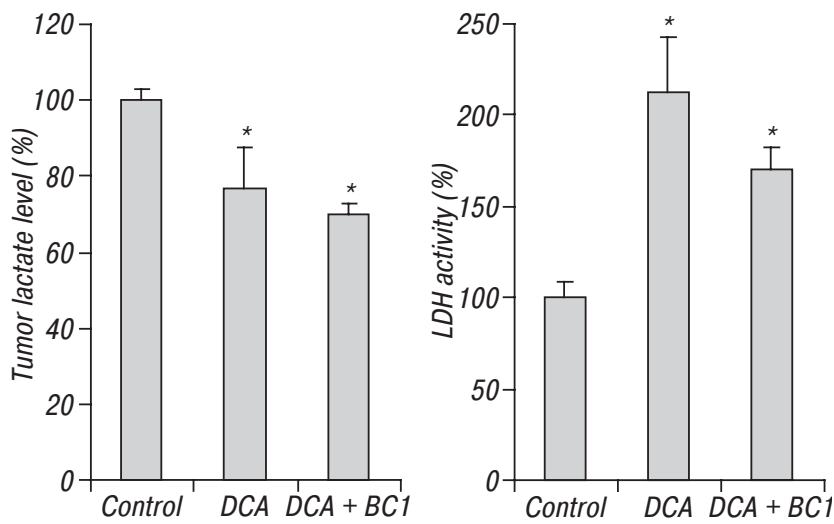

Fig. 1. Lactate level $(a)$ and LDH activity $(b)$ in tumor tissue of LLC/R9 bearing mice: control, DCA-treated and DCA + BC1treated groups. ${ }^{*} p<0.05$ compared to control

Our study has shown that administration of DCA alone or DCA in combination with BC1 resulted in significant increase of ROS production in tumor cells as well as normal cells involved in tumor process (TAM). High ROS production in tumor cells was manifested in enhancement of nitrosylation both heme proteins (with generation of NO-heme complexes) and non-heme proteins (with generation of dinitrosyl-iron complexes; DNIC). The latest play a key role in MtETC functioning.

As one may see on Fig. 2, administration of DCA alone or in combination with $\mathrm{BC} 1$ caused statistically significant elevation of DNIC level (Fig. 2, a) by $140 \%$ $(p<0.05)$ and $180 \%(p<0.01)$, as well as NO-heme complexes (Fig. $2, b)$ by $85 \%(p<0.05)$ and $43 \%$ $(p<0.05)$, respectively.
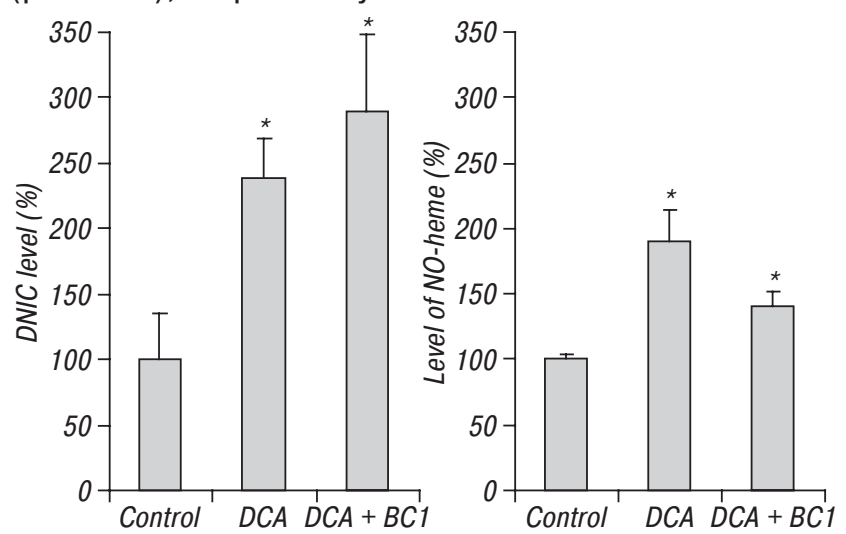

Fig. 2. Levels of DNIC (a) and NO-heme $(b)$ in tumor tissue of LLC/R9 bearing mice: control, DCA-treated and DCA + BC1treated groups. ${ }^{*} p<0.05$ compared to control

It is known that generation of $\mathrm{NO}-\mathrm{Fe}-\mathrm{S}$ clusters (DNIC) could lead to inactivation of Fe-S cluster proteins in MtETC, disturb coupling of oxidative phosphorylation with respiration and cause tumor cell death [25]. As it is shown on Fig. 3, significant increase of DNIC level in tumor tissue of mice after DCA administration did not lead to decrease of Fe-S cluster content, but at the same time Fe-S cluster content was 2 -fold lower $(p<0.01)$ in tumor tissue of mice treated with DCA and BC1 than that indices in control group of animals. These results have indicated that stimulation of ROS production induced by DCA administration did not cause MtETC dysfunction and had insignificant impact in anticancer activity of this agent. In contrary, stimulation of ROS production observed after combined administration of DCA and BC1 and decreased levels of Fe-S clusters made a contribution in the antitumor activity of combined therapy.

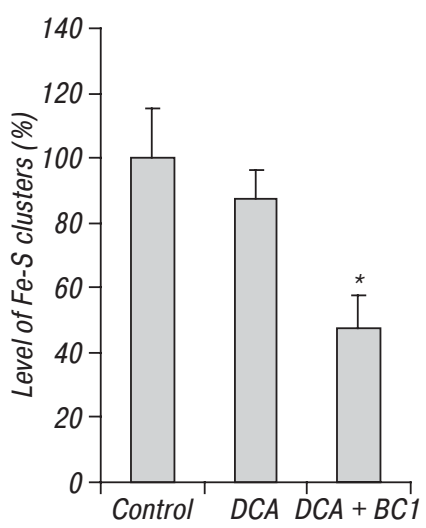

Fig. 3. Levels of Fe-S clusters in tumor tissue of LLC/R9 bearing mice: control, DCA-treated and DCA + BC1-treated groups. ${ }^{*} p<0.01$ compared to control

It is known that nitric oxide species appear in tumor tissue via its increased synthesis by mitochondrial NOsynthase (a constitutive isoform localized on internal mitochondrial membrane) and also by inducible NOsynthase of TAM.

TAMs develop from circulating monocytes recruited in the region of tumor growth because recognition and elimination of transformed cells is among physiologic functions of immune system [26]. Recruited mono- 
cytes in tumor lesion are capable to undergo functional diversification in two populations: macrophages with M2-phenotype which reveal proangiogenic action promoting tumor growth and progression; macrophages with M1-phenotype which participate in activation of antitumor immunity and reveal an expressed cytotoxic action against tumor cells [27]. Antitumor action of TAMs could be exerted by two mechanisms: phagocytic activity and ROS production.

Analysis of functional activity of TAM has shown significant increase of ROS production level by $241.0 \%$ $(p<0.01)$ in tumor tissue of mice after DCA administration at monoregimen compared to control animals (Fig. 4, c). The high level of ROS is determined by an expressed tendency to increased TAM counts in tumor tissue (Fig. 4, a) as well as activation of ROS production by TAM. DCA administration also led to activation of TAM phagocytic activity (Fig. $4, b$ ): in DCA-treated animals phagocytic activity of TAM was by $70.5 \%(p<0.05)$ higher than that in control mice. Such activation of cytotoxic activity of TAM evidences on significant impact of TAM on antitumor activity of DCA.
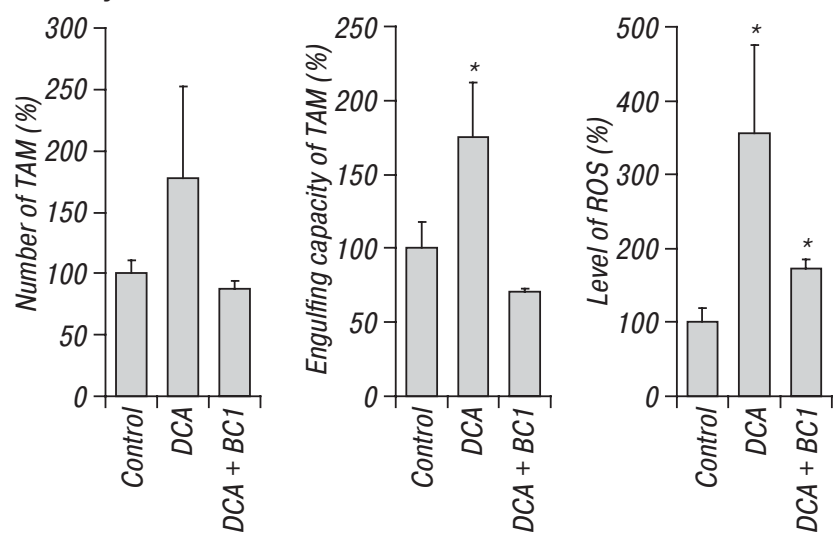

Fig. 4. Number of TAM $(a)$, their phagocytic activity $(b)$ and ROS production $(c)$ : control, DCA-treated and DCA + BC1-treated groups. ${ }^{*} p<0.05$ compared to control

It is necessary to note that an effect of DCA combined with $\mathrm{BC} 1$ toward counts and functional activity of TAM differed from that of DCA alone. As it is shown on Fig. 4, the combined therapy has no effect on TAM quantity and their phagocytic activity. After the combined therapy ROS production by TAM increased by $78 \%(p<0.05)$ compared to this index in control animals but was significantly lower compared to corresponding index in mice treated with DCA alone. So, this fact points on insignificant impact of cytotoxic activity of TAM in sufficiently high antitumor activity of DCA in combination with BC1.

In conclusion, the presented results have demonstrated that DCA exerts significant antitumor activity against Ehrlich carcinoma mainly via induced activation of cytotoxic activity of TAM. Antiangiogenic agent BC1 in combination with DCA significantly enhances antitumor activity of DCA via significant decrease of Fe$S$-containing protein level resulted from significant elevation of nitrosylation of these proteins.

\section{REFERENCES}

1. Warburg O. On the origin of cancer cells. Science 1956; 123: $309-14$

2. Jha MK, Suk K. Pyruvate dehydrogenase kinase as a potential therapeutic target for malignant gliomas. Brain Tumor Res Treat 2013; 1: 57-63.

3. Sutendra G, Michelakis ED. Pyruvate dehydrogenase kinase as a novel therapeutic target in oncology. Front Oncol 2013; 3: 38.

4. Kankotia S, Stacpoole PW. Dichloroacetate and cancer: new home for an orphan drug? Biochim Biophys Acta 2014; 1846: 617-29.

5. Papandreou I, Goliasova T, Denko NC. Anticancer drugs that target metabolism: is dichloroacetate the new paradigm? Int J Cancer 2011; 128: 1001-8.

6. Wong JY, Huggins GS, Debidda M, et al. Dichloroacetate induces apoptosis in endometrial cancer cells. Gynecol Oncol 2008; 109: 394-02.

7. Delaney LM, Ho N, Morrison J, et al. Dichloroacetate affects proliferation but not survival of human colorectal cancer cells. Apoptosis 2015; 20: 63-4.

8. Haugrud AB, Zhuang Y, Coppock JD, et al. Dichloroacetate enhances apoptotic cell death via oxidative damage and attenuates lactate production in metformin-treated breast cancer cells. Breast Cancer Res Treat 2014; 147: 539-50.

9. Michelakis ED, Webster L, Mackey JR. Dichloroacetate (DCA) as a potential metabolic-targeting therapy for cancer. Br J Cancer 2008; 99: 989-94.

10. Sun RC, Fadia M, Dahlstrom JE, et al. Reversal of the glycolytic phenotype by dichloroacetate inhibits metastatic breast cancer cell growth in vitro and in vivo. Breast Cancer Res Treat 2010; 120: 253-60.

11. Madhok BM, Yeluri S, Perry SL, et al. Dichloroacetate induces apoptosis and cell-cycle arrest in colorectal cancer cells. Br J Cancer 2010; 102: 1746-52.

12. Stockwin LH, Yu SX, Borgel S, et al. Sodium dichloroacetate selectively targets cells with defects in the mitochondrial ETC. Int J Cancer 2010; 127: 2510-9.

13. Anderson KM, Jajeh J, Guinan $P$, et al. In vitro effects of dichloroacetate and $\mathrm{CO}_{2}$ on hypoxic HeLa cells. Anticancer Res 2009; 29: 4579-88.

14. Yaromina A, Meyer S, Fabian C. Effects of three modifiers of glycolysis on ATP, lactate, hypoxia, and growth in human tumor cell lines in vivo. Strahlenther Onkol 2012; 188: 431-7.

15. Chu QS, Sangha R, Spratlin J, et al. A phase I openlabeled, single-arm, dose-escalation, study of dichloroacetate (DCA) in patients with advanced solid tumors. Invest New Drugs 2015; 33: 603-10.

16. Khan A, Marier D, Marsden E, et al. A novel form dichloroacetate therapy for patients with advanced cancer: a report of 3 cases. Altern Ther Health Med 2014; (Suppl 2): 21-8.

17. Kumar K, Wigfield S, Gee HE, et al. Dichloroacetate reverses the hypoxic adaptation to bevacizumab and enhances its antitumor effects in mouse xenografts. J Mol Med Berl 2013; 91: 749-58.

18. Solyanik GI, Fedorchuk AG, Pyaskovskaya ON, et al. Anticancer activity of aconitine-containing herbal extract BC1. Exp Oncol 2004; 26: 307-11.

19. Garmanchuk LV, Pyaskovskaya ON, Yahish YuV, et al. Influence of aconitine-containing herbal extract $\mathrm{BC} 1$ on proliferative and electrokinetic characteristics of endothelial cells. Exp Oncol 2005; 27: 262-6.

20. Proxorova MI. Biochemical methods (lipid and energy metabolism). Leningrad University, 1982.272 p. (in Russian).

21. Haitova RM. The use of flow cytometry to assess the functional activity of human immune system. Manual for physicians. Institute of Immunology 2001; 53 p. (in Russian). 
22. Pyaskovskaya ON, Boychuk IV, Fedorchuk AG, et al. Antitumor effects of dichloroacetate against Ehrlich carcinoma. Pharmacol Drug Toxicol 2014; 5: 73-9 (in Ukrainian).

23. Dasyukevich OI, Solyanik GI. Comparative study of anticancer efficacy of aconitine-containing agent $\mathrm{BC} 1$ against ascite and solid forms of Ehrlich's carcinoma. Exp Oncol 2007; 29: 317-9.

24. Lin G, Hill DK, Andrejeva G, Boult JKR, et al. Dichloroacetate induces autophagy in colorectal cancer cells and tumours. Br J Cancer 2014; 111: 375-85. doi: 10.1038/bjc.2014.281.
25. Pacher P, Beckman J, Liaudet L. Nitric oxide and peroxynitrite in health and disease. Physiol Rev 2007; 87: 315-24.

26. Mantovani A, Sica A, Allavena P, et al. Tumor-associated macrophages and the related myeloid-derived suppressor cells as a paradigm of the diversity of macrophage activation. Hum Immunol 2009; 70: 325-30.

27. Stout RD, Watkins SK, Suttles J. Functional plasticity of macrophages: in situ reprogramming of tumor-associated macrophages. Suttles J Leukoc Biol 2009; 86: 1105-9. 